\title{
MAFA: Multispectral Adaptive Fuzzy Algorithm for Edge Detection on MRI of Head Scan
}

\author{
Humera Tariq, $\mathrm{PhD}$ \\ Assistant Professor, \\ Department of Computer \\ Science, University of Karachi
}

\author{
Muhammad Shahbaz \\ Academic Researcher \\ University of Karachi
}

\author{
Humera \\ Assistant Professor \\ Department of Computer \\ Science, University of Karachi
}

\begin{abstract}
The purpose of this research is to propose a new Multispectral Adaptive Fuzzy Algorithm (MAFA) for edge detection in Magnetic Resonance Images (MRI). Edge detection is primary pre-segmentation process of MRI. Human structure is envisioned through 3-dimension images provided by MRI. MAFA, 40 Fuzzy Rules based algorithm, is proposed for edge detection because it is efficient in time consumption and processing calculations, effective in results and easier to use than other methods like Canny, Sobel, etcetera. Application of MAFA on 159 images produced sharper and clearer edges than other methods. Average time to process one image is 16 milliseconds which is $61 \%$ of time consumed by second best method.
\end{abstract}

\section{Keywords}

Fuzzy Algorithm, Edge Detection, MRI, Head Scans

\section{INTRODUCTION}

Image processing in the discourse of medical sciences have become substantially important to diagnose, deciding and executing surgeries, and ultimately treating today's complex diseases. There are number of techniques of medical images and technologies are available; for instance, X-rays, Ultra Sound, Computed Tomography (CT), Magnetic Resonance Image (MRI) etcetera. These techniques have different features along with their pros and cons. However, MRI is least harmful and best result oriented technique of nowadays. It is well known as non-ionizing, non-destructive, and non-invasive technology. Today's medical world heavily relies on MRI for imaging brains, backbone, and many other sensitive parts of the body. MRI technology, generally, uses three techniques for imaging which include Proton Density (PD), T1-weighted, and T2weighted. While scanning human head, MRI takes images in three angles which include axial, sagittal, and coronal. Whereas, axial images of head are taken from top to down, sagittal are left to right, and coronal are back to front.

Deformities are malformations of tissues in human body. So as to identify any deformity, segmenting and analyzing the structure of tissues i.e. brain structure in case of head imaging are essential steps. In order to segment any object and background of image, it is essential to detect edges. The process of segmenting objects existsbetween high-level and low-level image processing. The objecting process separates objects from images to understand the structure. This also helps removing unnecessary and irrelevant information from the image without compromising actual structure for better understanding of the matter in hand [1].Since, an edge is periphery between the actual object and background of image; which means accurately identifying edges are essential for understanding different features of the image and object such as shape, perimeter, and area [1]. Therefore, edge detection is important for effective result-oriented image processing. There are numerous methods of edge detection are available out which popular include Sobel, Fuzzy Logic, Robert Cross, and Canny [2].

Fuzzy Logic (FL) is one very effectualslant which helps solving problems based on particular logic[3]. Primarily, FL helps arriving at workable conclusion by depending on indistinct, indefinite, vague, and missing information [3] [4]. FL is workable at various stages, in multiples disciplines, and decision making processes[4]. Use of FL for edge detecting in digital images has been proposed by Abdallah and Ayman [5] Application of FL for edge detection in head scan has been proposed by Somasundaram and Ezhilarasan [6] [7].

The proposed application of FL for edge detection from digital images [5] are not robust for MRI head scanning. Moreover, proposed application of FL for edge detection [6] [7] is carrying 32 rules. More rules can be generated for better edge detection from digital images. This research explored new rules, Multispectral Adaptive Fuzzy Algorithm (MAFA) in extension with previous studies.

\section{METHODOLOGY}

This research is carried out under Scientific Paradigm of Research Philosophy [8]. This research paradigm supports research in computer sciences at all three positions of knowledge i.e. Ontology, Epistemology, and Methodology.This paradigm takes programs in parity with human mental processing, the ontology; defines computer science as study of empiricism and experimentalism, the methodology; and deduces and does experiments in order to obtain a priori and posteriori knowledge about the object under study, the epistemology. Since, this is scientific paradigm; therefore, it is objective research where knower (researcher) and known (knowledge) both have dichotomous relation.

There is two-steps methodical process to establish MAFA. First step comprises of Riddler's method for find the intensity threshold of image. Whereas, second step involves rules of FL for edge detecting from MRI of head scans.

\section{RESULTS AND DISCUSSION}

Data is analyzed following aforesaid two-steps process:-

\subsection{Riddler's Method}

Following steps are followed for executing Riddler's method:-

a. The average intensity value $\left(\mathrm{AIV}_{1}\right)$ of all pixels $(\mathrm{P})$ in the input image (II) is calculated using following formula:-

$$
A I V_{1}=\frac{\sum_{i=0}^{P-1} X_{i}}{P}
$$

Whereas, "P" is total number of pixels, "Xi" is intensity of one pixel. 
b. $\mathrm{AIV}_{1}$ is calculated to split the pixels of the input image i.e. $\mathrm{H}_{1}$ and $\mathrm{H}_{2}$ :-

$$
f\left(X_{i}\right) \in \begin{cases}H_{1} & \text { if }\left(X_{i}>A I V_{1}\right. \\ H_{2} & \text { otherwise }\end{cases}
$$

c. Average(AIV) of means of both halves $\mathrm{H}_{1}$ and $\mathrm{H}_{2}$ is calculated using following formula:-

$$
A I V=\frac{\frac{\sum_{i=0}^{p} H_{1}}{p\left(H_{1}\right)}+\frac{\sum_{i=0}^{p} H_{2}}{p\left(H_{2}\right)}}{2}
$$

Values ofp (H1) and p (H2) are numbers of all pixels in $\mathrm{H} 1$ and H2respectively. Step 2 and 3 continue until AIV $\approx$ AIV1.

d. The Final threshold value helps obtaining final binary image $\mathrm{II}_{\mathrm{b}}$ :-

$$
I I_{b}(X)=\left\{\begin{array}{lc}
1 & \text { if }(I I(X) \geq A I V \\
0 & \text { Otherwise }
\end{array}\right.
$$

\subsection{Fuzzy System}

A knowledge based method that is used to solve complex problems is called Fuzzy Systems [1] [3] [5] [7]. The same is used to detect edge of digital images where MRI characteristics will be the prior knowledge for applying FL. If-else rules are used to execute FL in any situation [3] [5][7]. Normally, FL consists of four main components which includefuzzifier, interface machine, knowledge base, and de-fuzzifier. Since, it is methodical process; where in first step i.e. fuzzifier, data is transformed into linguistic format which is called fuzzification. In second step, interface machine gets prior knowledge from knowledge base for data processing. In third step, de-fuzzifier transforms data results into crisp data which is called defuzzification. The process is illustrated as figure -1 .

\subsection{Fuzzy Rules}

There are 40 Fuzzy Rules (FR) are framed and methodically applied on digital images. In this context, digital mask of size 3 $\mathrm{x} 3$ is designed. The same digital mask is applied on binary input image(IIb), illustrated in figure - 2. In one IIb, "PIX" is current pixel while PIX1, PIX2, PIX3, PIX4, PIX5, PIX6, PIX7, and PIX8 are neighbor pixels.

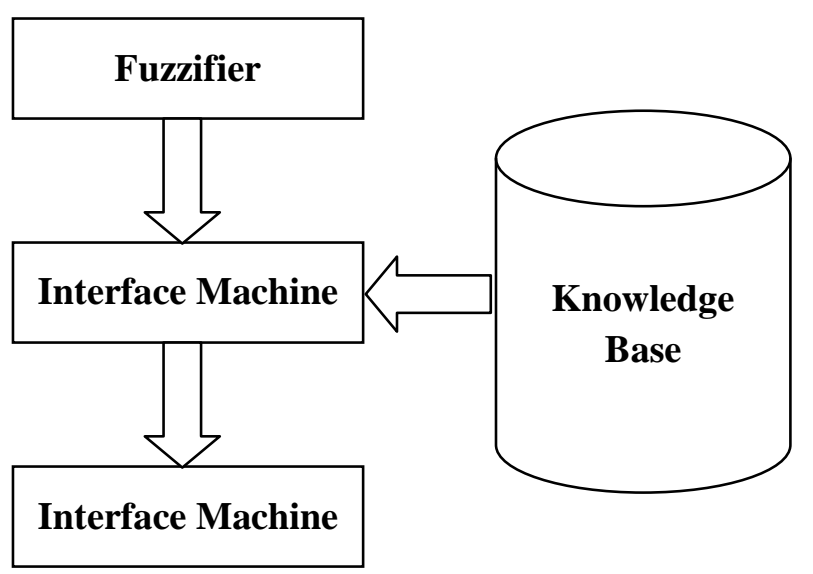

Figure 1: Fuzzy system - a methodical process

\begin{tabular}{|c|c|c|}
\hline $\mathrm{PIX}_{1}$ & $\mathrm{PIX}_{2}$ & $\mathrm{PIX}_{3}$ \\
\hline $\mathrm{PIX}_{8}$ & $\mathrm{PIX}$ & $\mathrm{PIX}_{4}$ \\
\hline $\mathrm{PIX}_{7}$ & $\mathrm{PIX}_{6}$ & $\mathrm{PIX}_{5}$ \\
\hline
\end{tabular}

Figure 2: Mask on digital image

Current and its neighboring pixels arethe base for applying fuzzy rules. During execution of FL based on If-else function, bright and dark pixels pass 0 and 1 respectively. Following are $40 \mathrm{FL}$

\begin{tabular}{|c|c|c|}
\hline SR & Fuzzy Rule & Mask \\
\hline & $\mathrm{PIX}_{1}=1$ & \\
\hline & $\mathrm{PIX}_{2} \& \mathrm{PIX}_{3} \& \mathrm{PIX}_{4} \& \mathrm{PIX}_{5} \& \mathrm{PIX}_{6} \& \mathrm{PIX}_{7} \& \mathrm{PIX}_{8}$ & \\
\hline & $\mathrm{PIX}_{2}=1$ & \\
\hline & $\mathrm{PIX}_{1} \& \mathrm{PIX}_{3} \& \mathrm{PIX}_{4} \& \mathrm{PIX}_{5} \& \mathrm{PIX}_{6} \& \mathrm{PIX}_{7} \& \mathrm{PIX}_{8}$ & \\
\hline & $\mathrm{PIX}_{3}=1$ & \\
\hline & $\mathrm{PIX}_{1} \& \mathrm{PIX}_{2} \& \mathrm{PIX}_{4} \& \mathrm{PIX}_{5} \& \mathrm{PIX}_{6} \& \mathrm{PIX}_{7} \& \mathrm{PIX}_{8}$ & \\
\hline & $\mathrm{PIX}_{4}=1$ & \\
\hline & $\mathrm{PIX}_{1} \& \mathrm{PIX}_{2} \& \mathrm{PIX}_{3} \& \mathrm{PIX}_{5} \& \mathrm{PIX}_{6} \& \mathrm{PIX}_{7} \& \mathrm{PIX}_{8}$ & \\
\hline & $\mathrm{PIX}_{5}=1$ & \\
\hline & $\mathrm{PIX}_{1} \& \mathrm{PIX}_{2} \& \mathrm{PIX}_{3} \& \mathrm{PIX}_{4} \& \mathrm{PIX}_{6} \& \mathrm{PIX}_{7} \& \mathrm{PIX}_{8}$ & \\
\hline & $\mathrm{PIX}_{6}=1$ & \\
\hline & $\begin{array}{c}\mathrm{PIX}_{1} \& \mathrm{PIX}_{2} \& \mathrm{PIX}_{3} \& \mathrm{PIX}_{4} \& \mathrm{PIX}_{5} \& \mathrm{PIX}_{7} \& \mathrm{PIX}_{8} \\
=0\end{array}$ & \\
\hline & & \\
\hline & & \\
\hline
\end{tabular}
based on same concept:-

Table 1: 40 Fuzzy Rules 

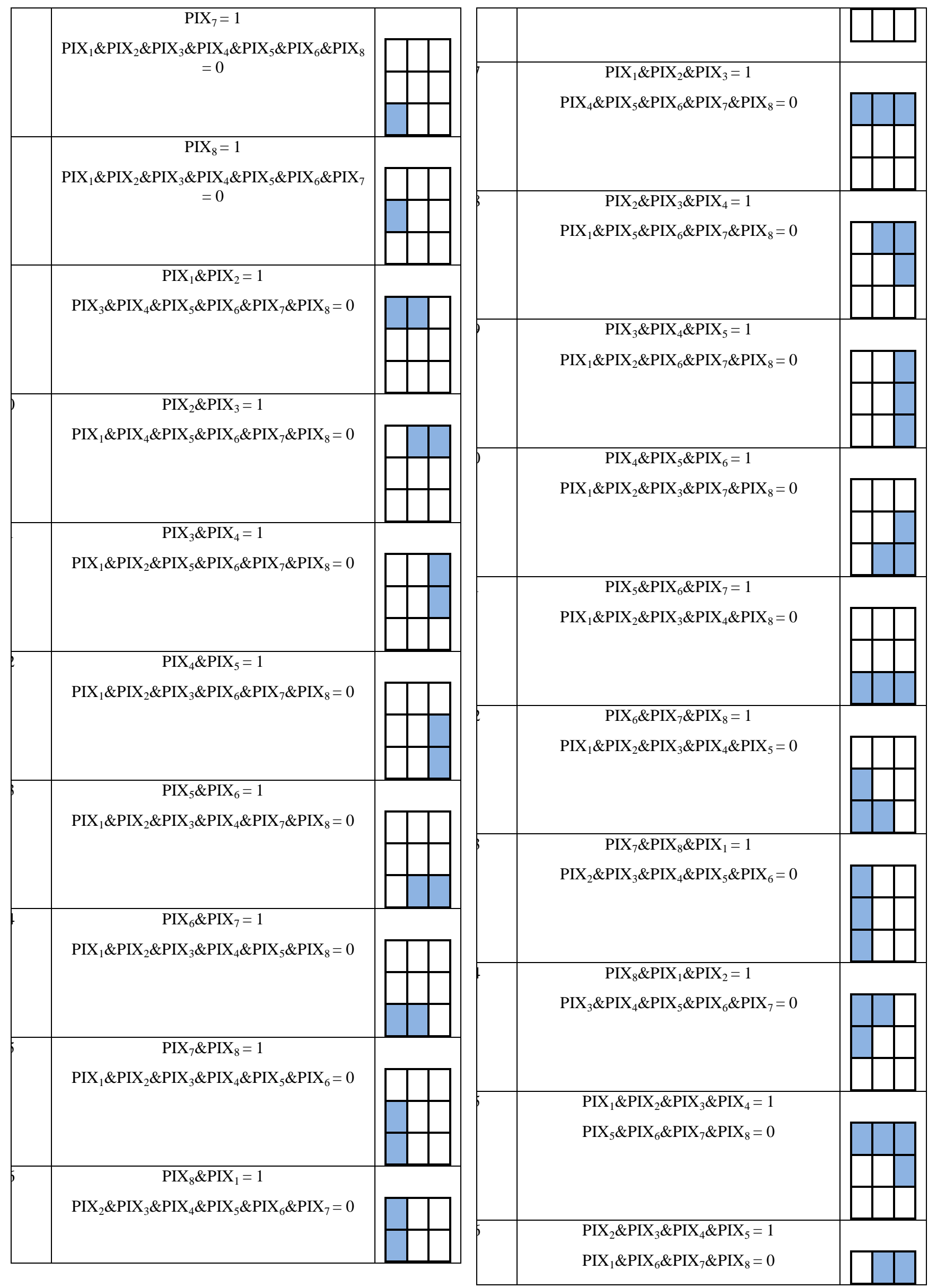

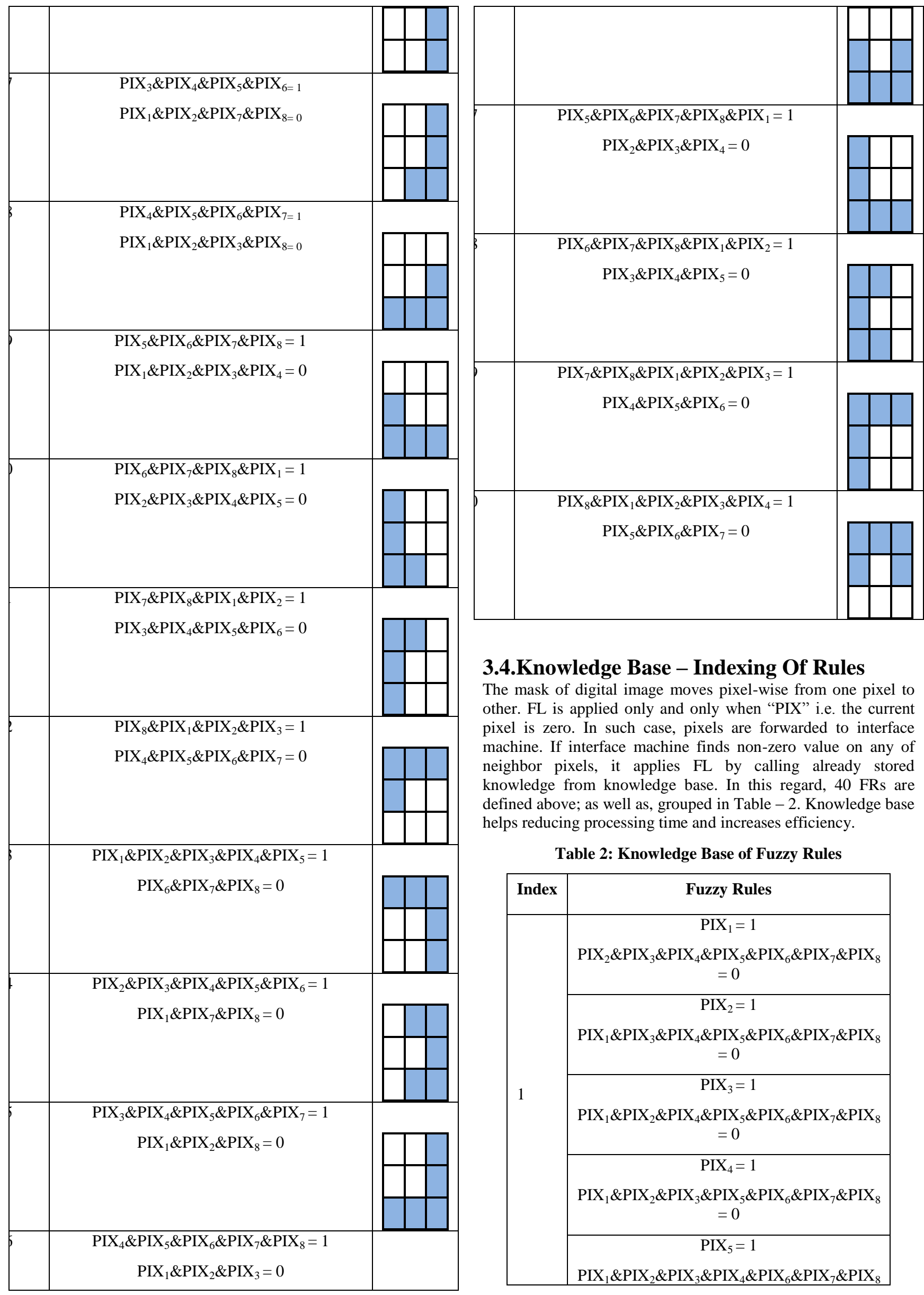

\subsection{Knowledge Base - Indexing Of Rules}

The mask of digital image moves pixel-wise from one pixel to other. FL is applied only and only when "PIX" i.e. the current pixel is zero. In such case, pixels are forwarded to interface machine. If interface machine finds non-zero value on any of neighbor pixels, it applies FL by calling already stored knowledge from knowledge base. In this regard, 40 FRs are defined above; as well as, grouped in Table - 2. Knowledge base helps reducing processing time and increases efficiency.

Table 2: Knowledge Base of Fuzzy Rules

\begin{tabular}{|c|c|}
\hline Index & Fuzzy Rules \\
\hline \multirow{1}{*}{1} & $\mathrm{PIX}_{1}=1$ \\
& $\mathrm{PIX}_{2} \& \mathrm{PIX}_{3} \& \mathrm{PIX}_{4} \& \mathrm{PIX}_{5} \& \mathrm{PIX}_{6} \& \mathrm{PIX}_{7} \& \mathrm{PIX}_{8}$ \\
& $=0$ \\
\cline { 2 - 3 } & $\mathrm{PIX}_{2}=1$ \\
& $\mathrm{PIX}_{1} \& \mathrm{PIX}_{3} \& \mathrm{PIX}_{4} \& \mathrm{PIX}_{5} \& \mathrm{PIX}_{6} \& \mathrm{PIX}_{7} \& \mathrm{PIX}_{8}$ \\
& $=0$ \\
\cline { 2 - 3 } & $\mathrm{PIX}_{1} \& \mathrm{PIX}_{2} \& \mathrm{PIX}_{4} \& \mathrm{PIX}_{5} \& \mathrm{PIX}_{6} \& \mathrm{PIX}_{7} \& \mathrm{PIX}_{8}$ \\
& $=0$ \\
\cline { 2 - 3 } & $\mathrm{PIX}_{4}=1$ \\
& $\mathrm{PIX}_{1} \& \mathrm{PIX}_{2} \& \mathrm{PIX}_{3} \& \mathrm{PIX}_{5} \& \mathrm{PIX}_{6} \& \mathrm{PIX}_{7} \& \mathrm{PIX}_{8}$ \\
& $=0$ \\
\cline { 2 - 3 } & $\mathrm{PIX}_{5}=1$ \\
& $\mathrm{PIX}_{1} \& \mathrm{PIX}_{2} \& \mathrm{PIX}_{3} \& \mathrm{PIX}_{4} \& \mathrm{PIX}_{6} \& \mathrm{PIX}_{7} \& \mathrm{PIX}_{8}$ \\
\hline
\end{tabular}




\begin{tabular}{|c|c|}
\hline & $=0$ \\
\hline & $\begin{array}{c}\mathrm{PIX}_{6}=1 \\
\mathrm{PIX}_{1} \& \mathrm{PIX}_{2} \& \mathrm{PIX}_{3} \& \mathrm{PIX}_{4} \& \mathrm{PIX}_{5} \& \mathrm{PIX}_{7} \& \mathrm{PIX}_{8} \\
=0\end{array}$ \\
\hline & $\begin{array}{c}\mathrm{PIX}_{7}=1 \\
\mathrm{PIX}_{1} \& \mathrm{PIX}_{2} \& \mathrm{PIX}_{3} \& \mathrm{PIX}_{4} \& \mathrm{PIX}_{5} \& \mathrm{PIX}_{6} \& \mathrm{PIX}_{8} \\
=0\end{array}$ \\
\hline & $\begin{array}{c}\mathrm{PIX}_{8}=1 \\
\mathrm{PIX}_{1} \& \mathrm{PIX}_{2} \& \mathrm{PIX}_{3} \& \mathrm{PIX}_{4} \& \mathrm{PIX}_{5} \& \mathrm{PIX}_{6} \& \mathrm{PIX}_{7} \\
=0\end{array}$ \\
\hline \multirow{8}{*}{2} & $\begin{array}{c}\text { PIX }_{1} \& \mathrm{PIX}_{2}=1 \\
\mathrm{PIX}_{3} \& \mathrm{PIX}_{4} \& \mathrm{PIX}_{5} \& \mathrm{PIX}_{6} \& \mathrm{PIX}_{7} \& \mathrm{PIX}_{8}=0\end{array}$ \\
\hline & $\begin{array}{c}\mathrm{PIX}_{2} \& \mathrm{PIX}_{3}=1 \\
\mathrm{PIX}_{1} \& \mathrm{PIX}_{4} \& \mathrm{PIX}_{5} \& \mathrm{PIX}_{6} \& \mathrm{PIX}_{7} \& \mathrm{PIX}_{8}=0\end{array}$ \\
\hline & $\begin{array}{c}\mathrm{PIX}_{3} \& \mathrm{PIX}_{4}=1 \\
\mathrm{PIX}_{1} \& \mathrm{PIX}_{2} \& \mathrm{PIX}_{5} \& \mathrm{PIX}_{6} \& \mathrm{PIX}_{7} \& \mathrm{PIX}_{8}=0\end{array}$ \\
\hline & $\begin{array}{c}\mathrm{PIX}_{4} \& \mathrm{PIX}_{5}=1 \\
\mathrm{PIX}_{1} \& \mathrm{PIX}_{2} \& \mathrm{PIX}_{3} \& \mathrm{PIX}_{6} \& \mathrm{PIX}_{7} \& \mathrm{PIX}_{8}=0\end{array}$ \\
\hline & $\begin{array}{c}\mathrm{PIX}_{5} \& \mathrm{PIX}_{6}=1 \\
\mathrm{PIX}_{1} \& \mathrm{PIX}_{2} \& \mathrm{PIX}_{3} \& \mathrm{PIX}_{4} \& \mathrm{PIX}_{7} \& \mathrm{PIX}_{8}=0\end{array}$ \\
\hline & $\begin{array}{c}\mathrm{PIX}_{6} \& \mathrm{PIX}_{7}=1 \\
\mathrm{PIX}_{1} \& \mathrm{PIX}_{2} \& \mathrm{PIX}_{3} \& \mathrm{PIX}_{4} \& \mathrm{PIX}_{5} \& \mathrm{PIX}_{8}=0\end{array}$ \\
\hline & $\begin{array}{c}\mathrm{PIX}_{7} \& \mathrm{PIX}_{8}=1 \\
\mathrm{PIX}_{1} \& \mathrm{PIX}_{2} \& \mathrm{PIX}_{3} \& \mathrm{PIX}_{4} \& \mathrm{PIX}_{5} \& \mathrm{PIX}_{6}=0\end{array}$ \\
\hline & $\begin{array}{c}\text { PIX }_{8} \& \mathrm{PIX}_{1}=1 \\
\mathrm{PIX}_{2} \& \mathrm{PIX}_{3} \& \mathrm{PIX}_{4} \& \mathrm{PIX}_{5} \& \mathrm{PIX}_{6} \& \mathrm{PIX}_{7}=0\end{array}$ \\
\hline \multirow{7}{*}{3} & $\begin{array}{c}\mathrm{PIX}_{1} \& \mathrm{PIX}_{2} \& \mathrm{PIX}_{3}=1 \\
\mathrm{PIX}_{4} \& \mathrm{PIX}_{5} \& \mathrm{PIX}_{6} \& \mathrm{PIX}_{7} \& \mathrm{PIX}_{8}=0\end{array}$ \\
\hline & $\begin{array}{c}\mathrm{PIX}_{2} \& \mathrm{PIX}_{3} \& \mathrm{PIX}_{4}=1 \\
\mathrm{PIX}_{1} \& \mathrm{PIX}_{5} \& \mathrm{PIX}_{6} \& \mathrm{PIX}_{7} \& \mathrm{PIX}_{8}=0\end{array}$ \\
\hline & $\begin{array}{c}\mathrm{PIX}_{3} \& \mathrm{PIX}_{4} \& \mathrm{PIX}_{5}=1 \\
\mathrm{PIX}_{1} \& \mathrm{PIX}_{2} \& \mathrm{PIX}_{6} \& \mathrm{PIX}_{7} \& \mathrm{PIX}_{8}=0\end{array}$ \\
\hline & $\begin{array}{c}\mathrm{PIX}_{4} \& \mathrm{PIX}_{5} \& \mathrm{PIX}_{6}=1 \\
\mathrm{PIX}_{1} \& \mathrm{PIX}_{2} \& \mathrm{PIX}_{3} \& \mathrm{PIX}_{7} \& \mathrm{PIX}_{8}=0\end{array}$ \\
\hline & $\begin{array}{c}\mathrm{PIX}_{5} \& \mathrm{PIX}_{6} \& \mathrm{PIX}_{7}=1 \\
\mathrm{PIX}_{1} \& \mathrm{PIX}_{2} \& \mathrm{PIX}_{3} \& \mathrm{PIX}_{4} \& \mathrm{PIX}_{8}=0\end{array}$ \\
\hline & $\begin{array}{c}\mathrm{PIX}_{6} \& \mathrm{PIX}_{7} \& \mathrm{PIX}_{8}=1 \\
\mathrm{PIX}_{1} \& \mathrm{PIX}_{2} \& \mathrm{PIX}_{3} \& \mathrm{PIX}_{4} \& \mathrm{PIX}_{5}=0\end{array}$ \\
\hline & $\begin{array}{c}\mathrm{PIX}_{7} \& \mathrm{PIX}_{8} \& \mathrm{PIX}_{1}=1 \\
\mathrm{PIX}_{2} \& \mathrm{PIX}_{3} \& \mathrm{PIX}_{4} \& \mathrm{PIX}_{5} \& \mathrm{PIX}_{6}=0\end{array}$ \\
\hline 4 & $\begin{array}{c}\mathrm{PIX}_{8} \& \mathrm{PIX}_{1} \& \mathrm{PIX}_{2}=1 \\
\mathrm{PIX}_{3} \& \mathrm{PIX}_{4} \& \mathrm{PIX}_{5} \& \mathrm{PIX}_{6} \& \mathrm{PIX}_{7}=0\end{array}$ \\
\hline
\end{tabular}

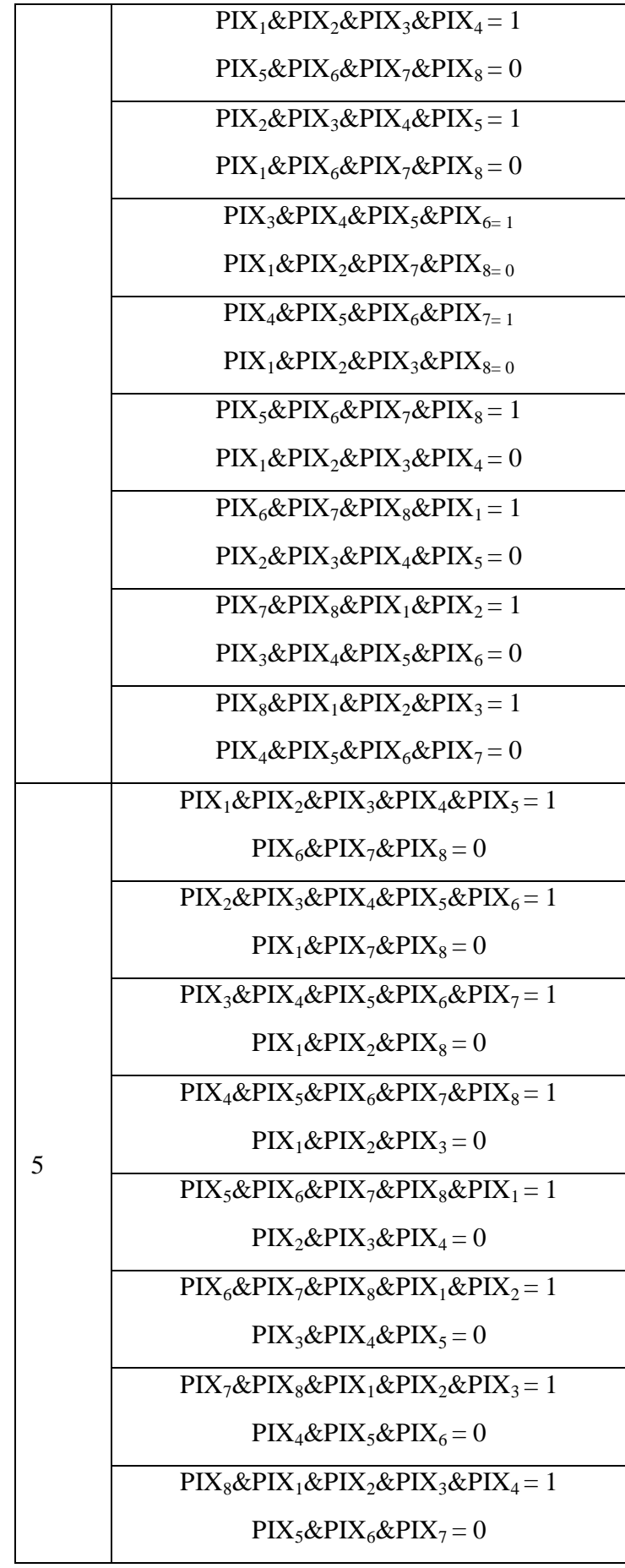

\subsection{Efficiency of Mafa}

MAFA is comparatively more efficient than other popular tools like Sobel and Canny. Total 172 randomly selected images were masked out of which 159 could be transferred to interface machine. Rest 13 images could not fuzzified because of current pixel "PIX" was non-zero. Therefore, output i.e. de-fuzzification was carried out from 159 images. Table -3 is showing comparative analysis of efficiency of MAFA:- 
Table 3: Efficiency Comparison

\begin{tabular}{|c|c|c|c|}
\hline Criteria & Canny & Sobel & MAFA \\
\hline Calculations & 4284 & 3757 & 3444 \\
\hline $\begin{array}{c}\text { Processing Time } \\
\text { (milliseconds) }\end{array}$ & 240 & 26 & 16 \\
\hline
\end{tabular}

\subsection{Edge Detection Quality}

Edge detection of MAFA is finer in quality and better in results. Table -4 is illustrating MAFA based edge detection images.

Table 4: MAFA quality

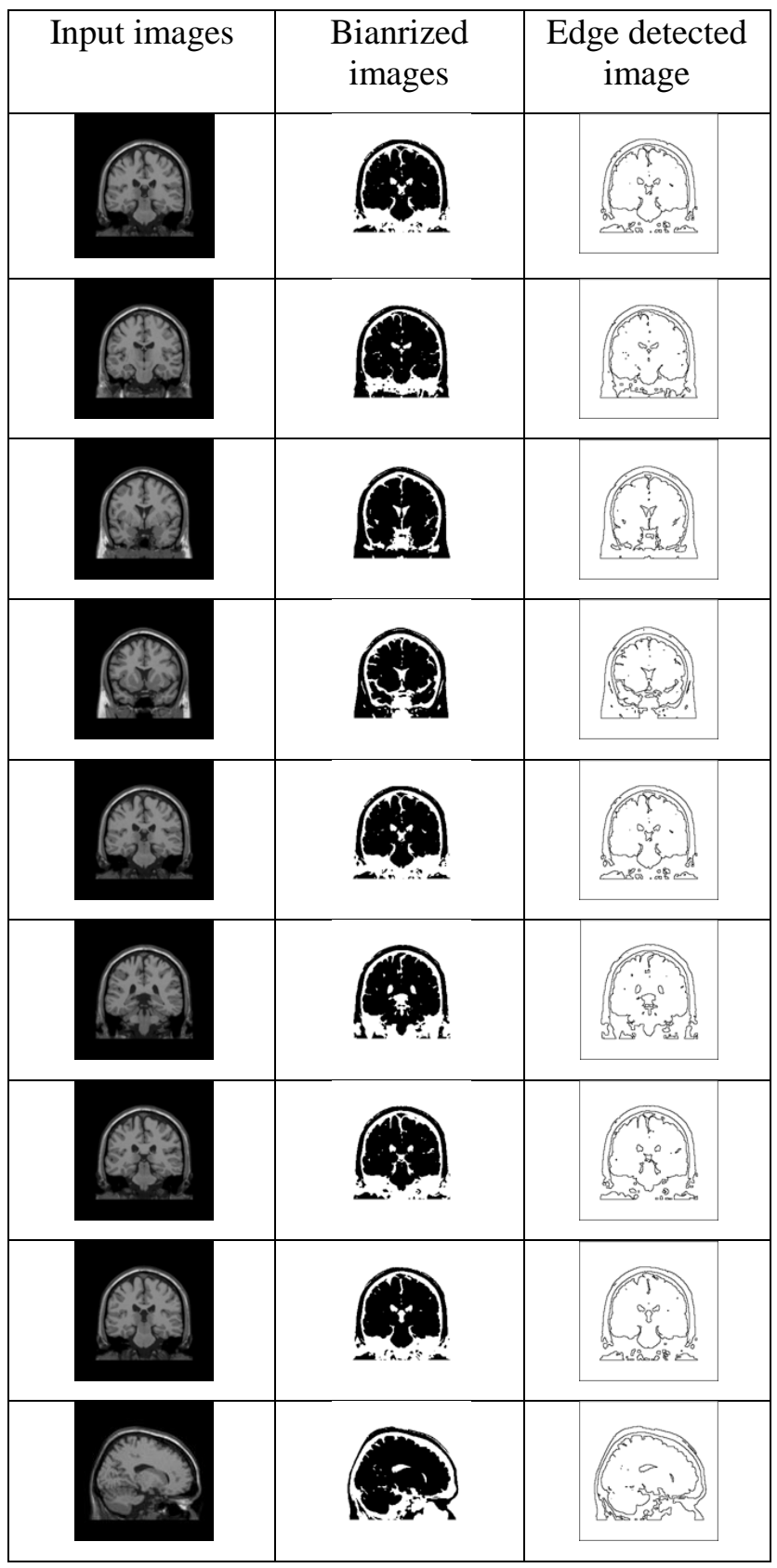

\subsection{Edge Detection Comparison}

In order to compare output-based performance of MAFA with Sobel and Canny, 3 outputs are shown in Table -5 .

Table 5: Comparison of MAFA results with other tests

\begin{tabular}{|c|c|c|c|c|c|c|}
\hline Sr. & A & B & $\mathrm{C}$ & $\mathrm{D}$ & $\mathrm{E}$ & $\mathrm{F}$ \\
\hline 1 & & & & & & \\
\hline 2 & & & & & & \\
\hline 3 & & & & & & \\
\hline
\end{tabular}

Legend key: $\mathrm{A}=$ original image, $\mathrm{B}=$ Canny, $\mathrm{C}=$ Prewitt, $\mathrm{D}=$ Robert, $\mathrm{E}=$ Sobel, $\mathrm{F}=\mathrm{MAFA}$

\section{CONCLUSION}

One robust method, MAFA, for edge detection of digital images especially for MRI of head scans is experimented which is less complex to understand and apply, more efficient in time consumption, better and clearer results, less in number of calculations by processor as compare to other methods like Canny, Sobel etcetera. MAFA is proposed for effectively and efficiently segmenting objects from digital images especially MRI.

\section{REFERENCES}

[1] Gonzalez, R. C., Woods, R. E., Digital Image Processing, 3rd ed. Upper Saddle River, New Jersey, United States of America: Prentice Hall, 2002.

[2] Umbaugh, S. E., Digital Image Processing and Analysis: Human and Computer Vision Applications with CVIPtools, 2nd ed. Boca Raton, Florida, United States of America: CRC Press, 2010.

[3] Dernoncourt, F. (2013) http://www.francky.me. [Online]. http://www.francky.me/doc/course/fuzzy_logic.pdf

[4] Hellmann,M. (2015) https://ecs.syr.edu. [Online]. https://ecs.syr.edu/faculty/Tetley/ELE\%20516\%20CIS\%20 543/Fuzzy\%20Logic/Fuzzy\%20Logic\%20Introduction.pdf

[5] Alshennawy, A. A., Aly, A. A., "Edge Detection in Digital Images Using Fuzzy Logic Technique," World Academy of Science, Engineering and Technology, vol. 51, pp. 178186, 2009.

[6] Ezhilarasan, K., Somasundaram, K., "Edge Detection in MRI of Head Scans Using Fuzzy Logic," in IEEE International Conference on Advanced Communication Control and Computing Technologies (ICACCCT), 2012, pp. 131-135.

[7] Ezhilarasan, K., Somasundaram K., "Edge Detection using Fuzzy Logic and Thresholding," in National Conference on Signal and lmnge Processing, 2012, pp. 157-160.

[8] Raymond, T., "Philosophy of Computer Science: Introduction to the Special Issue," Minds and Machines, vol. 17, no. 2, pp. 129-133, 2007. 